\title{
Chemorheology of Photopolymerizable Acrylates Using a Modified Boltzmann Sigmoidal Model
}

\author{
B. J. LOVE, ${ }^{1,2,3}$ F. PIGUET RUINET, ${ }^{4}$ F. TEYSSANDIER ${ }^{3}$ \\ ${ }^{1}$ Department of Materials Science and Engineering, University of Michigan, Ann Arbor, \\ Michigan 48109 \\ ${ }^{2}$ Department of Biomedical Engineering, University of Michigan, Ann Arbor, Michigan 48109 \\ ${ }^{3}$ Department of Biological and Materials Sciences (Dentistry), University of Michigan, Ann Arbor, Michigan 48109 \\ ${ }^{4}$ Department of Materials Science and Engineering, Virginia Polytechnic Institute and State University, \\ Blacksburg, Virginia 24061
}

Received 24 April 2008; revised 19 August 2008; accepted 20 August 2008

DOI: 10.1002 / polb.21563

Published online in Wiley InterScience (www.interscience.wiley.com).

\begin{abstract}
Experiments were conducted to evaluate the influence of ambient photoconversion on rheology for a range of photopolymerizable urethane dimethacrylate (UDMA) resins containing varying amounts of three comonomers including 1,6 hexane diol-dimethacrylate (HDDMA), an alkoxylated cyclohexane dimethanol diacrylate monomer (CD-582), and hydroxyethyl methacrylate (HEMA). Experiments were performed both as a function of composition and time-dependent dose varying the intensity using a photorheometer. A semilog-based sigmoidal model allowed the determination of four physical model parameters to define the relationship between reaction kinetics and its dynamic influence on viscosity. We have observed induction times and viscosity changes associated with the model that shows a trend in reaction kinetics in the following order from most to least reactive: UDMA > CD582 > HDDMA > HEMA. With increasing amounts of reactive diluent included in the formulation, the kinetics of reaction was more sluggish. The value of this sigmoidal model is that it could help define formulation and process conditions most likely to control crosslinking to maximize dimensional stability or other thermophysical properties. () 2008 Wiley Periodicals, Inc. J Polym Sci Part B: Polym Phys 46: 2319-2325, 2008

Keywords: acrylates; conversion; crosslinking; modeling; photopolymerization; rheology; sigmoidal
\end{abstract}

\section{INTRODUCTION}

Cure advancement of thermosetting resins has been of significant interest in establishing molecular parameters linked with describing the gel edu)

Correspondence to: B. J. Love (E-mail: bjlove@umich.

Journal of Polymer Science: Part B: Polymer Physics, Vol. 46, 2319-2325 (2008) (C) 2008 Wiley Periodicals, Inc. point,${ }^{1}$ the induction time before gelation or the working time, and network architecture, all which are related to bulk chemical formulation and reactivity. $^{2,3}$ Understanding cure advancement has been the driving force behind establishing process parameters for thermosets such as lamination temperatures and pressures necessary to trigger or block larger scale flow ${ }^{4,5}$ or to control shrinkage. ${ }^{6-8}$ Extensive efforts over a long time have been made to determine the kinetics of conversion 
in a range of reactive resins and their diffusional dependence on the thermodynamic activation energy of conversion by evaluating reaction kinetics as a function of temperature. ${ }^{9-13}$ An excellent recent review has related conversion and the capacity for molecular diffusion for several common polymerization schemes. ${ }^{14}$ And a number of both theoretical and empirical models of viscosity advancement were summarized by Halley and Mackay. ${ }^{15}$ Publications have also gauged both reactive stability and defined flow and processing characteristics for reactive injection molding resins and other flowing resins. ${ }^{16-18}$

The link between conversion and rheology has garnered our own interest in the stability of reactive dispersions, ${ }^{19,20}$ and conversion of highly crosslinkable photocurable polymers where induced viscosity changes occur very quickly. ${ }^{20,21}$ We have used conceptually simple cure advancement models such as the power law model seen in eq 1 below:

$$
\log \eta(t)=\log \eta_{0}+n t
$$

where $\eta(t)$ is the time-dependent viscosity, $\eta_{0}$ is a pregelled initial viscosity at which crosslinking is initiated, $n$ is related to the rapidity of the viscosity rise, and $t$ is the time. We analyzed both published data on curing epoxy resins including filled and unfilled resins, and photopolymerized resins where the kinetics of cure are more rapid. ${ }^{21,22}$

This power law rheology model, while conceptually simple, can deviate significantly as network density rises. We considered whether other nonlinear analytical models could more accurately follow dynamic viscosity changes induced by crosslinking. ${ }^{23-25}$ Others have used a Boltzmann sigmoidal model to describe other viscous fluids undergoing a setting reaction including starches, ${ }^{26}$ siloxane based impression compounds, ${ }^{25}$ and other gels. In our adapted model, we fitted the following Boltzmann sigmoidal function in eq 2

$$
\log \eta(t)=\log \eta_{\infty}+\frac{\log \left(\eta_{0}\right)-\log \left(\eta_{\infty}\right)}{1+e^{\left(\frac{t-t_{0}}{\Delta t}\right)}}
$$

where $\eta_{0}$ and $\eta_{\infty}$ are the viscosities before illumination and after network formation at long times of illumination. The induction time, $t_{0}$, corresponded to the time necessary a change viscosity from the initial viscosity $\log \eta_{0}$ to $\left(\log \eta_{0}+\log \eta_{\infty}\right) /$ 2 , and $\Delta t$ corresponds with the period associated with the sigmoidal transition region as viscosity deviates from $\eta_{0}$ in the semiloglinear regime. All these parameters vary with illumination inten- sity, temperature, oxygen content, and other factors that regulate the kinetics of polymerization.

We conducted experiments to evaluate the influence of ambient photoconversion on the rheology of urethane dimethacrylate (UDMA) resins mixed with varying amounts of three comonomers including: hexane diol-dimethacrylate (HDDMA), an alkoxylated cyclohexane dimethanol diacrylate (CD-582 or CHDMDA), and hydroxyethyl methacrylate (HEMA). Urethane dimethacrylate is a dental base monomer in composite formulations, ${ }^{7,27-30}$ and is commonly mixed with other reactive diluents. Experiments were performed varying composition and time dependent dose varying the intensity using a photorheometer following Schmidt et al. ${ }^{31}$ The results were analyzed and comparisons were made as a function of the parametric analysis.

\section{EXPERIMENTAL}

\section{Resin Preparation}

Urethane dimethacrylate, UDMA (ESSTECH, Essington, PA), was mixed with one of the following monomers: 1,6-hexanediol-dimethacrylate, HDDMA (ESSTECH, Essington, PA), alkoxylated cyclohexane dimethanol diacrylate resin, CHDMDA (CD-582 Sartomer, PA), and hydroxyethyl methacrylate (HEMA; Polysciences, Warrington, $\mathrm{PA}$ ) using weight fractions of $0.5,0.7$, and 0.85 in UDMA. The formulation included 0.5 w/w\% of both $N, N$-Dimethyl- $p$-toluidine (Aldrich) and camphorquinone (Aldrich) as part of the photosensitizer package. Each mixed formulation was shaken overnight and wrapped in aluminum foil before use.

\section{Rheology Experiments}

Dynamic viscosity advancement during photopolymerization was obtained using an ARES rheometer from TA instruments, equipped with a broadband EFOS Novacure N2000 Spotcure UV lamp assembly following Schmidt et al. ${ }^{31}$ This lamp is housed $0.7 \mathrm{~m}$ from the rheometer with a flexible light pipe to illuminate the stage. The beam was calibrated using a light meter supplied by TA Instruments to ensure consistent illumination intensity across the plate before initiating each photorheology experiment. Each experiment initially measured $\eta_{0}$ in the dark before the trigger time when the shutter opened illuminating the stage at intensities between 1 and $5 \mathrm{~W} / \mathrm{cm}^{2}$. 


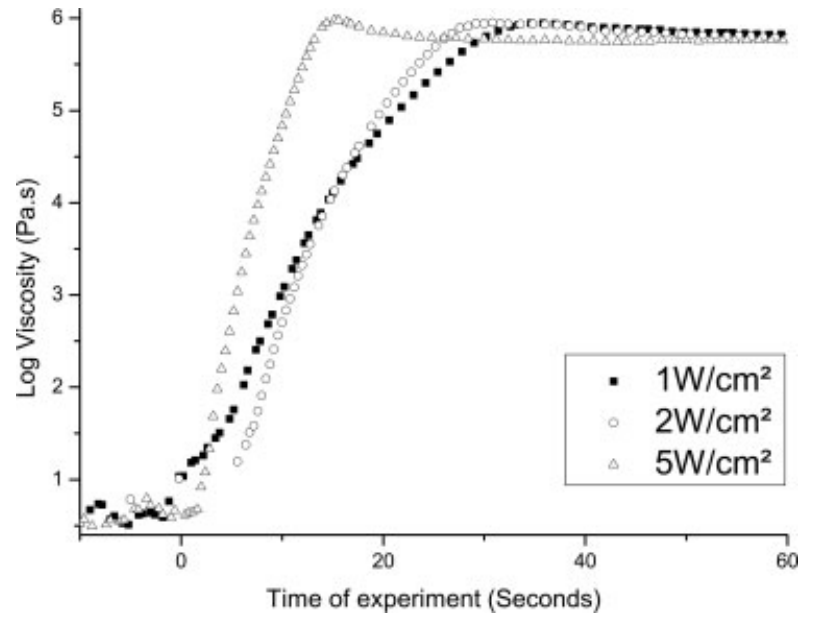

Figure 1. Dynamic viscosity with fluence for a 70\%UDMA/30\%HEMA mixture.

The distance between the lamp and sample was deemed sufficient to discount ambient heating as a factor affecting polymerization. In situ polymerization occurred between an aluminum substrate and a transparent acrylic substrate which was part of the optical path. Parallel plate rheometry in continuous shear was chosen to avoid optical influences using a more accurate cone and plate assembly. This selection also allowed us the potential to repolish and reuse the disks. The rheometer was located in a room held nominally at $27^{\circ} \mathrm{C}$.

The gap between the two substrates was set to $0.3 \mathrm{~mm}$. Three drops of each liquid monomer mixture were placed on the aluminum substrate and then contacted with the $2 \mathrm{~cm}$ diameter acrylic substrate. An engine drove the lower part of the rheometer and separate measurements of torque and frequency $(20 \mathrm{rad} / \mathrm{s})$ resolved the transient polymer viscosity. Experiments were conducted at 1,2 , and $5 \mathrm{~W} / \mathrm{cm}^{2}$ to yield two replicates although individual experiments were analyzed. Experiments in which the initial viscosity before illumination was outside the range of the component viscosities were discarded outright.

\section{Analysis}

The data were collected with TA Orchestrator software and exported into a database. Once the datasets were constructed in the form of instantaneous viscosity with time, these data were loaded into Origin (Microcal) from which statistical determinations of sigmoidal plot parameters were found. With $\log \eta_{0}(\mathrm{~Pa} \mathrm{~s})$ ranging between 0 and 1 , and $\eta_{\infty}$ fixed at $1 \times 10^{6} \mathrm{~Pa} \mathrm{~s}$, the sigmoidal plot analysis yielded two parameters including the induction time, $t_{0}$ and $\Delta t$, the time constant inversely proportional to slope of the dynamic rise in viscosity.

\section{RESULTS AND DISCUSSION}

Figure 1 shows the dynamic viscosity profile during photopolymerization of a 70\%UDMA/ 30\%HEMA mixture using three different illumination intensities. As expected, with rising fluence, the speed of conversion was increased. The scale of the slope change was sufficiently large in comparison with other studies of curing resins that the logarithmic analysis was justified. Both the asymptotic limits in viscosity, $\left(\eta_{0}\right.$ and $\left.\eta_{\infty}\right)$ are essentially the same, the network structure is similar, even if polymerized with different light intensity.

The sigmoidal fits for the data above with log $\eta_{0}(\mathrm{~Pa} \mathrm{~s})$ and $\log \eta_{\infty}(\mathrm{Pa} \mathrm{s})$ fixed at 0.4 and 6 are shown in Figures 2-4 (straight lines) for $1 \mathrm{~W} / \mathrm{cm}^{2}$ (Fig. 2), $2 \mathrm{~W} / \mathrm{cm}^{2}$ (Fig. 3), and $5 \mathrm{~W} / \mathrm{cm}^{2}$ (Fig. 4) for a mixture of $70 \%$ UDMA/30\%HEMA. Similar trends were observed and characterized for the other reactive diluents. Each curve was modeled up to the asymptotic limit. The drop off in viscosity seen in Figure 1 is attributed to slip of the bonding disks at high conversion and viscosity.

Table 1 includes sigmoidal fit parameters for individual experiments varying the composition and type of reactive diluent, and intensity. Replicate experiments confirmed the trends observed. Included in the analysis are the determined

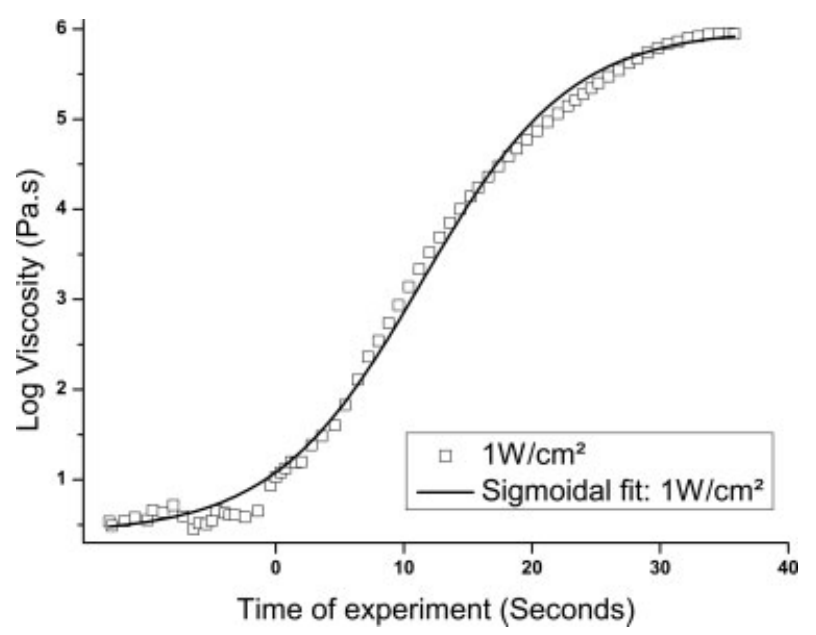

Figure 2. Dynamic viscosity illuminated at $1 \mathrm{~W} / \mathrm{cm}^{2}$ along with sigmoidal fit for a 70\%UDMA/30\%HEMA mixture. 


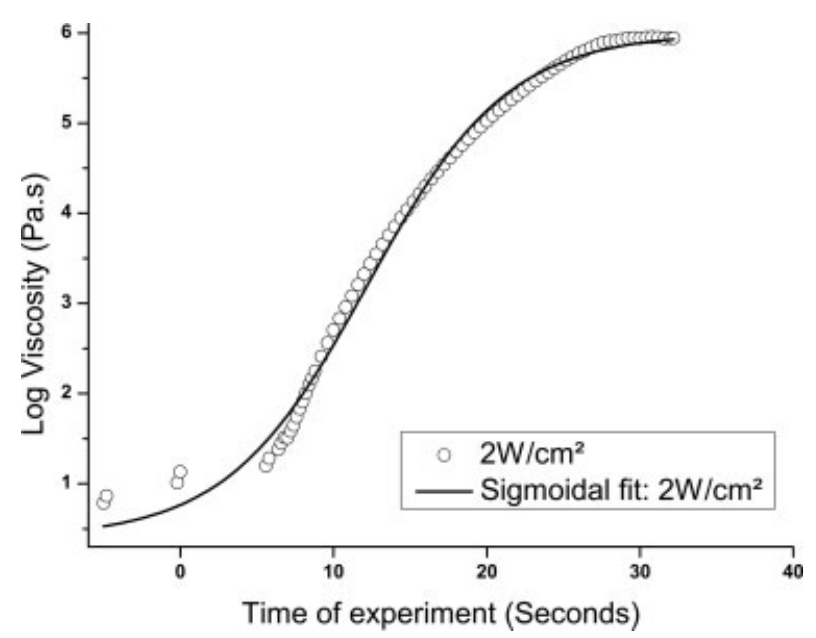

Figure 3. Dynamic viscosity illuminated at $2 \mathrm{~W} / \mathrm{cm}^{2}$ along with the sigmoidal fit 70\%UDMA/30\%HEMA mixture.

induction time $t_{0}$, and $\Delta t$, the time period which is inversely related to the slope associated with the log-linear dynamic viscosity curve.

The sigmoidal model successfully represents the dynamics of viscosity during photopolymerization. In terms of trends, Table 1 shows that reactivity generally increased with higher UDMA content and higher intensity illumination, as evidenced by lower induction times and shorter periods $(\Delta t)$ associated with the rate of viscosity rise.

Based on intensity alone, the induction time should depend on the time necessary to grow a sufficient number of radicals to initiate large-scale crosslinking. The trend of shorter illumination times to induce this transition with higher intensity is readily observable, although initiation and propagation are expected to occur at different rates.

Adding less-reactive diluents such as HEMA extends the induction period by as much as $30 \mathrm{~s}$ at $1 \mathrm{~W} / \mathrm{cm}^{2}$. UDMA has the fastest reaction kinetics in terms of combined radical formation and propagation of the resins evaluated. Here, we confirm other published work by Assumption and Mathias $^{29}$ as well as our own work. ${ }^{21}$

\section{Rate of Viscosity Rise and $\Delta t$, the Period Associated with Viscosity Rise}

The slope of the viscosity rise region rises with both higher illumination intensity and UDMA content as evidenced by a shorter time constant, $\Delta t$. Adding more diluent stunts the rate of poly- merization. An example is shown in Figure 5 plotting the time constant associated with viscosity rise as a function of illumination intensity mixed with 50\% with each comonomer. The trend in slope at each condition is generally conserved, with UDMA the most reactive, followed by CD582, HDDMA, and HEMA with the longest periods.

Comparing different diluents, CD-582 induces conversion faster than HDDMA and HEMA, and a higher slope associated with the shorter time constant, $\Delta t$ in the Boltzmann sigmoidal model. Mixtures of CD-582 and UDMA were the most reactive. With other published efforts suggesting that a lower conversion rate is tied to lower polymerization shrinkage, ${ }^{32-34}$ it is possible that polymerization shrinkage could be controlled with larger concentrations of less reactive monomers in the mix, and lower polymerization intensity, although that was not part of our study. Other recent work using a new type of in situ shrinkage measurement suggests that the correlation between conversion rate and shrinkage may be murky. ${ }^{35-39}$ Future mechanistic understanding of resin chemistry, evolving network structure, and functional properties based on variations in the polymerization protocol are needed. It is also possible that other functional attributes such as monomer extraction potential could also be affected by variations in the network structure induced by different photopolymerization protocols or formulations. ${ }^{40}$

HEMA was the most sluggish of the monomers we evaluated in triggering the induction. In

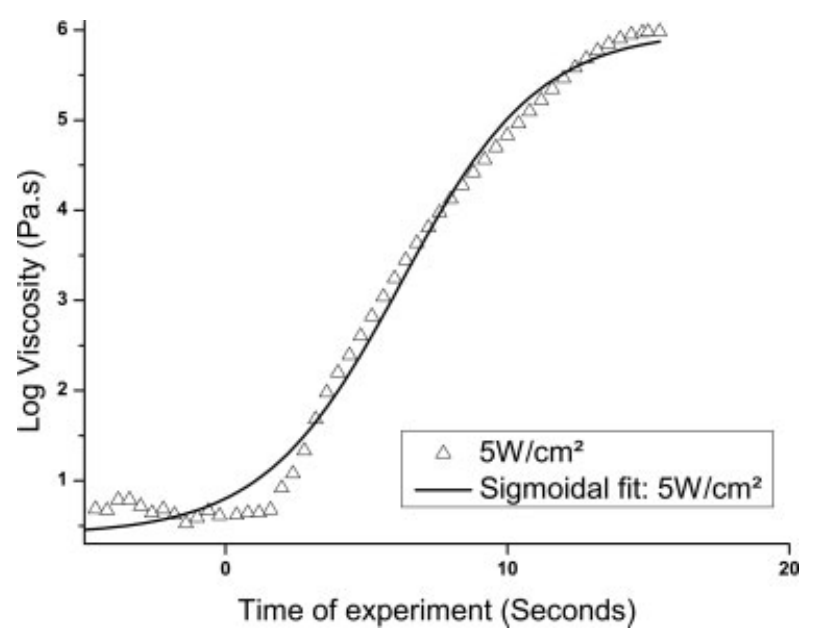

Figure 4. Dynamic viscosity illuminated at $5 \mathrm{~W} / \mathrm{cm}^{2}$ along with the sigmoidal fit $70 \%$ UDMA/30\%HEMA mixture.

Journal of Polymer Science: Part B: Polymer Physics DOI $10.1002 /$ polb 
Table 1. Tabulated Parameters for the Boltzmann Sigmoidal Analysis of UDMA-Based Resins

\begin{tabular}{|c|c|c|c|c|c|}
\hline & \multicolumn{2}{|c|}{ Comonomer } & \multirow[b]{2}{*}{$\begin{array}{l}\text { Induction } \\
\text { Time } t_{0}(\mathrm{~s})\end{array}$} & \multirow[b]{2}{*}{$\Delta t(\mathrm{~s})$} & \multirow[b]{2}{*}{$\begin{array}{c}\text { Correlation } \\
\text { Coefficient } R^{2}\end{array}$} \\
\hline & $\begin{array}{c}\text { Mass } \\
\text { Fraction }\end{array}$ & $\begin{array}{l}\text { Intensity } \\
\left(\mathrm{W} / \mathrm{cm}^{2}\right)\end{array}$ & & & \\
\hline \multirow[t]{9}{*}{ HEMA } & 0.15 & 1 & 8.01 & 3.40 & 0.984 \\
\hline & & 2 & 4.32 & 2.97 & 0.990 \\
\hline & & 5 & 3.01 & 1.70 & 0.985 \\
\hline & 0.3 & 1 & 11.31 & 5.75 & 0.996 \\
\hline & & 2 & 12.1 & 4.64 & 0.995 \\
\hline & & 5 & 6.23 & 2.45 & 0.992 \\
\hline & 0.5 & 1 & 40.79 & 9.54 & 0.990 \\
\hline & & 2 & 26.46 & 5.98 & 0.983 \\
\hline & & 5 & 16.87 & 4.22 & 0.982 \\
\hline \multirow[t]{9}{*}{ HDDMA } & 0.15 & 1 & 10.24 & 3.17 & 0.956 \\
\hline & & 2 & 7.02 & 2.98 & 0.977 \\
\hline & & 5 & 4.04 & 1.70 & 0.978 \\
\hline & 0.3 & 1 & 12.49 & 3.22 & 0.959 \\
\hline & & 2 & 9.3 & 2.33 & 0.962 \\
\hline & & 5 & 5.9 & 1.67 & 0.974 \\
\hline & 0.5 & 1 & 17.33 & 4.04 & 0.979 \\
\hline & & 2 & 11.07 & 3.07 & 0.980 \\
\hline & & 5 & 6.18 & 2.06 & 0.990 \\
\hline \multirow[t]{9}{*}{ CD582 } & 0.15 & 1 & 7.77 & 2.99 & 0.959 \\
\hline & & 2 & 5.08 & 2.18 & 0.966 \\
\hline & & 5 & 3.28 & 1.47 & 0.971 \\
\hline & 0.3 & 1 & 9.5 & 3.19 & 0.977 \\
\hline & & 2 & 6.54 & 2.51 & 0.972 \\
\hline & & 5 & 4.84 & 1.21 & 0.968 \\
\hline & 0.5 & 1 & 9.54 & 3.58 & 0.986 \\
\hline & & 2 & 6.41 & 2.06 & 0.980 \\
\hline & & 5 & 3.88 & 2.01 & 0.992 \\
\hline
\end{tabular}

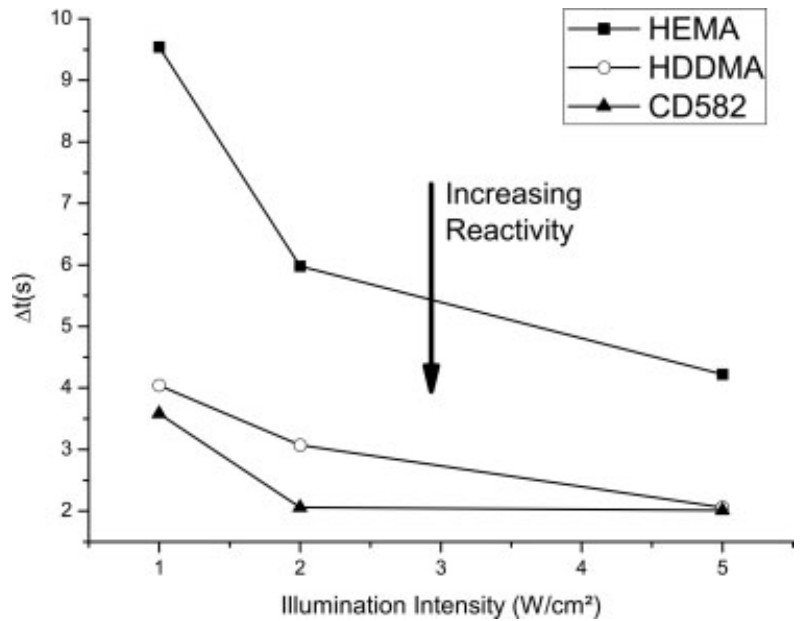

Figure 5. Comparison of the period of viscosity rise, $\Delta t$, in the sigmoidal model with fluence for UDMA resins mixed at $50 \% \mathrm{w} / \mathrm{w}$ with different comonomers. addition, HEMA at $0.3 \mathrm{wt}$ fraction exhibits a crossover in the induction time between samples illuminated at 1 and $2 \mathrm{~W} / \mathrm{cm}^{2}$. Since these are single measurements, we attribute this behavior to experimental error in both the sample construction and processing. No other formulations showed this same crossover effect but HEMA's sluggishness was clear.

In linking the photorheology results determined here to the broader polymerization literature, one long-range goal is to relate simpler phenomenological models that yield physically relevant parameters such as the rate of viscosity rise to molecular interpretations of conversion rates and diffusion limitations with increased conversion. Many papers have related dynamic conversion both in terms of thermodynamics and viscosity for a range of polymerizable reactions. ${ }^{12,14,31,41-44}$ With new measurements of 
photodynamically induced dynamic viscosity now available, the ability to link viscosity changes to conversion and chemical reaction kinetics models may lead to a larger fundamental understanding of how dose and polymerization rate affect structure.

If structure is influenced by polymerization rate, controlling it in photocrosslinked hydrogels could also affect functional release of therapeutic agents formulated into the structure. ${ }^{45-47}$ This type of model could be applied to colloidal or soft gels that cause changes in rheology upon gelation, ${ }^{26,43}$ or in biochemistry where polymerization of misfolded proteins in solution ${ }^{48}$ has a driving force for agglomeration and changing the dispersion structure as well.

\section{CONCLUSIONS}

An adapted sigmoidal model was used to analyze $\eta(t)$ arising from photopolymerization of UDMA mixtures varying both formulation and illumination intensity. We compared UDMA mixed with HDDMA, HEMA, and an experimental resin derived from alkoxylated polyethylene glycol dimethacrylate, CD-582. Two physical parameters came out of the analysis that relate to conversion including an induction time needed to trigger a 50\% conversion in $\log \eta$, and a time constant associated with the slope of the logarithmic viscosity rise.

One value of this model is that it accurately represents phenomenological viscosity changes arising due to photopolymerization. The model could also be adapted to other resin systems where there is a dynamic viscosity change due to changing solution or dispersion structure. ${ }^{49}$

We acknowledge Virginia Tech internal support mechanisms and fellowships from the Institut des Sciences et Techniques de l'Ingenieur de Lyon for both Mr PiguetRuinet and Mr Teyssandier. We also acknowledge Dr. Herve Marand who graciously hosted us in the laboratory where the rheometer was located.

\section{REFERENCES AND NOTES}

1. Madbouly, S. A.; Ougizawa, T. J Macromol Sci Phys 2004, B43, 655-670.

2. Gaur, B.; Rai, J. S. P. Polym Plast Technol Eng 2006, 45, 197-203.

3. Chiu, H. T.; Tsai, P. A.; Cheng, T. C. J Mater Eng Perform 2006, 15, 81-87.

4. Farrar, D. F.; Rose, J. Biomaterials 2001, 22, 3005-3013.
5. Nicholas, M. K. D.; Waters, M. G. J.; Holford, K. M.; Adusei, G. J Mater Sci Mater Med 2007, 18, 1407-1412.

6. Andrzejewska, E.; Socha, E.; Andrzejewski, M. Polymer 2006, 47, 6513-6523.

7. Floyd, C. J. E.; Dickens, S. H. Dent Mater 2006, 22, 1143-1149.

8. Gatti, A.; Rastelli, A. N. S.; Ribeiro, S. J. L.; Messaddeq, Y.; Bagnato, V. S. J Thermal Anal Calorim 2007, 87, 631-634.

9. Yang, Y. S.; Suspene, L. Polym Eng Sci 1991, 31, 321-332.

10. Laza, J. M.; Vilas, J. L.; Rodriguez, M.; Garay, M. T.; Mijangos, F.; Leon, L. M. J Appl Polym Sci 2002, 83, 57-65.

11. Laza, J. M.; Vilas, J. L.; Mijangos, F.; Rodriguez, M.; Leon, L. M. J Appl Polym Sci 2005, 98, 818-824.

12. Ryan, M. E.; Dutta, A. Polymer 1979, 20, 203-206.

13. Malkin, A. Y. Polym Eng Sci 1980, 20, 1035-1044.

14. Achilias, D. S. Macromol Theory Simul 2007, 16, 319-347.

15. Halley, P. J.; Mackay, M. E. Polym Eng Sci 1996, 36, 593-609.

16. Bullions, T. A.; McGrath, J. E.; Loos, A. C. Polym Eng Sci 2002, 42, 2182-2192.

17. Derose, A. N.; Yuan, M.; Osswald, T. A.; Castro, J. M. Polym Plast Technol Eng 2002, 41, 383-405.

18. Cheng, K. C.; Chiu, W. Y.; Hsieh, K. H.; Ma, C. C. M. J Mater Sci 1994, 29, 721-727.

19. Dolez, P. I.; Goff, A.; Love, B. J Separation Sci Technol 2002, 37, 2007-2019.

20. Love, B. J Particulate Sci Technol 2004, 22, 285-290.

21. Piguet-Ruinet, F.; Love, B. J. J Appl Polym Sci 2008, 107, 1523-1529.

22. Love, B. J.; Piguet-Ruinet, F. J Appl Polym Sci 2007, 106, 3605-3609.

23. Kim, K. J.; Kim, B. K. J Appl Polym Sci 1993, 48, 981-986.

24. Uhniat, M.; Zemlak, M.; John, J.; Szulc, R. Polimery 2003, 48, 709-713.

25. Berg, J. C.; Johnson, G. H.; Lepe, X.; Adan-Plaza, S. J Prosthetic Dentistry 2003, 90, 150-161.

26. Israkarn, K.; Hongsprabhas, P.; Hongsprabhas, P. Carbohydr Polym 2007, 68, 314-322.

27. Tanimoto, Y.; Hayakawa, T.; Nemoto, K. J Biomed Mater Res B Appl Biomat 2005, 72B, 310-315.

28. Dickens, S. H.; Stansbury, J. W.; Choi, K. M.; Floyd, C. J. E. Macromolecules 2003, 36, 6043-6053.

29. Assumption, H. J.; Mathias, L. J. Polymer 2003, 44, 5131-5136.

30. Sideridou, I.; Tserki, V.; Papanastasiou, G. Biomaterials 2002, 23, 1819-1829.

31. Schmidt, L. E.; Leterrier, Y.; Vesin, J.-M.; Wilhelm, M.; Manson, J.-A. Macromol Mater Eng 2005, 290, 1115-1124.

32. Feng, L.; Suh, B. I. J Biomed Mater Res B Appl Biomater 2006, 78B, 63-69.

33. Feng, L.; Suh, B. I. J Biomed Mater Res B Appl Biomater 2006, 76B, 196-202.

Journal of Polymer Science: Part B: Polymer Physics DOI $10.1002 /$ polb 
34. Lu, B.; Xiao, P.; Sun, M. Z.; Nie, J. J Appl Polym Sci 2007, 104, 1126-1130.

35. Lu, H.; Stansbury, J. W.; Bowman, C. N. J Dent Res 2005, 84, 822-826.

36. Lu, H.; Stansbury, J. W.; Bowman, C. N. Dent Mater 2004, 20, 979-986.

37. Lu, H.; Stansbury, J. W.; Dickens, S. H.; Eichmiller, F. C.; Bowman, C. N. J Mater Sci Mater Med 2004, 15, 1097-1103.

38. Lu, H.; Stansbury, J. W.; Dickens, S. H.; Eichmiller, F. C.; Bowman, C. N. J Biomed Mater Res B Appl Biomater 2004, 71B, 206-213.

39. Stansbury, J. W.; Trujillo-Lemon, M.; Lu, H.; Ding, X. Z.; Lin, Y.; Ge, J. H. Dent Mater 2005, 21, 56-67.

40. Vaubert, V. M.; Moon, P. C.; Love, B. J. J Biomed Mater Res 1999, 48, 5-8.

41. Sun, Y.; Zhang, Z.; Wong, C. P. Macromol Mater Eng 2005, 290, 1204-1212.
42. Achilias, D. S.; Sideridou, I. D. Macromolecules 2004, 37, 4254-4265.

43. Kulichikhin, S. G.; Malkin, A. Y.; Polushkina, O. M.; Kulichikhin, V. G. Polym Eng Sci 1997, 37, 13311338.

44. Dutta, A.; Ryan, M. E. J Appl Polym Sci 1979, 24, 635-649.

45. Leach, J. B.; Bivens, K. A.; Collins, C. N.; Schmidt, C. E. J Biomed Mater Res A 2004, 70A, 74-82.

46. Leach, J. B.; Bivens, K. A.; Patrick, C. W.; Schmidt, C. E. Biotechnol Bioeng 2003, 82, 578589.

47. Leach, J. B.; Schmidt, C. E. Biomaterials 2005, 26, 125-135.

48. Wang, X. A.; Smith, D. R.; Jones, J. W.; Chapman, M. R. J Biol Chem 2007, 282, 3713-3719.

49. Wen, M.; Scriven, L. E.; McCormick, A. V. Macromolecules 2003, 36, 4151-4159. 